\title{
Pleuroparenchymal Fibroelastosis Associated with Crohn's Disease: A New Aetiology?
}

\author{
Carolina Amado ${ }^{1}$, Pedro Gonçalo Ferreira ${ }^{2}$ \\ ${ }^{1}$ Internal Medicine Department, Centro Hospitalar do Baixo Vouga, Aveiro, Portugal \\ ${ }^{2}$ Pulmonology Department, Centro Hospitalar do Baixo Vouga, Aveiro, Portugal
}

Doi: 10.12890/2020_002017 - European Journal of Case Reports in Internal Medicine - ๑ EFIM 2020

Received: $21 / 09 / 2020$

Accepted: 04/11/2020

Published: $25 / 11 / 2020$

How to cite this article: Amado C, Ferreira PG. Pleuroparenchymal fibroelastosis associated with Chron's disease: a new aetiology? EJCRIM 2020;7: doi:10.12890/2020_002017.

Conflicts of Interests: The Authors declare that there are no competing interests.

This article is licensed under a Commons Attribution Non-Commercial 4.0 License

\section{ABSTRACT}

Pleuroparenchymal fibroelastosis (PPFE) is a relatively rare interstitial lung disease (ILD) consisting of elastofibrosis involving the subpleural parenchyma and visceral pleura with an upper lobe predominance. It can be idiopathic or associated with some forms of autoimmune disease. The authors describe the case of a 78-year-old woman with a previous diagnosis of Crohn's disease (CD). She presented with a protracted respiratory infection (with no significant history of previous infections), and underwent high-resolution chest computerized tomography that eventually showed alterations compatible with PPFE. After exclusion of other possible underlying causes, a possible link to CD was considered. Follow-up investigation at 12 months showed clinical and radiological stability and also stable lung function under treatment with hydroxychloroquine and prednisolone. This is the first report of PPFE in the context of CD. Future studies will be important to further investigate this pathological association and its prognostic implications.

\section{LEARNING POINTS}

- Pleuroparenchymal fibroelastosis (PPFE) is an uncommon interstitial lung disease consisting of elastofibrosis involving the subpleural parenchyma and visceral pleura with an upper lobe predominance.

- PPFE can be idiopathic or associated with several conditions including autoimmune diseases and may be a direct extra-intestinal manifestation of Crohn's disease (CD).

- The main disease patterns of PPFE in the context of CD are organizing pneumonia, non-specific interstitial pneumonia, granulomatous interstitial lung disease, pulmonary eosinophilia and sterile necrobiotic nodules.

\section{KEYWORDS}

Pleuroparenchymal fibroelastosis, Crohn's disease, interstitial lung disease

\section{INTRODUCTION}

Pleuroparenchymal fibroelastosis (PPFE) is a relatively rare interstitial lung disease (ILD) consisting of elastofibrosis involving the subpleural parenchyma and visceral pleura with an upper lobe predominance ${ }^{[1]}$. It can idiopathic or associated with several conditions including some forms of autoimmune disease ${ }^{[1,2]}$. Crohn's disease (CD), in its turn, can be associated with ILD ${ }^{[3,4]}$ whether drug-related or not. In the context of $\mathrm{CD}$, the main associated disease patterns are organizing pneumonia, non-specific interstitial pneumonia, granulomatous interstitial lung disease, pulmonary eosinophilia and sterile necrobiotic nodules ${ }^{[5]}$. 


\section{CASE DESCRIPTION}

The authors present the case of a 78-year-old woman with CD under treatment with mesalazine (formerly in combination with azathioprine, the only prior drug). She did not have a history of previous respiratory illness, relevant inhalational exposures (tobacco, organic antigens or inorganic dust) or a family history of ILD. The patient had a normal level of immunocompetence, with no history of cancer or connective tissue disease. She had not previously undergone organ transplantation or radio- or chemotherapy. In the context of a protracted respiratory infection (with no significant history of previous infections), she underwent high-resolution chest computerized tomography (HRCT) that eventually showed fibrotic pleural thickening of the upper lobes and some reticulation and irregular opacities on the adjacent subpleural parenchyma (Fig. 1).
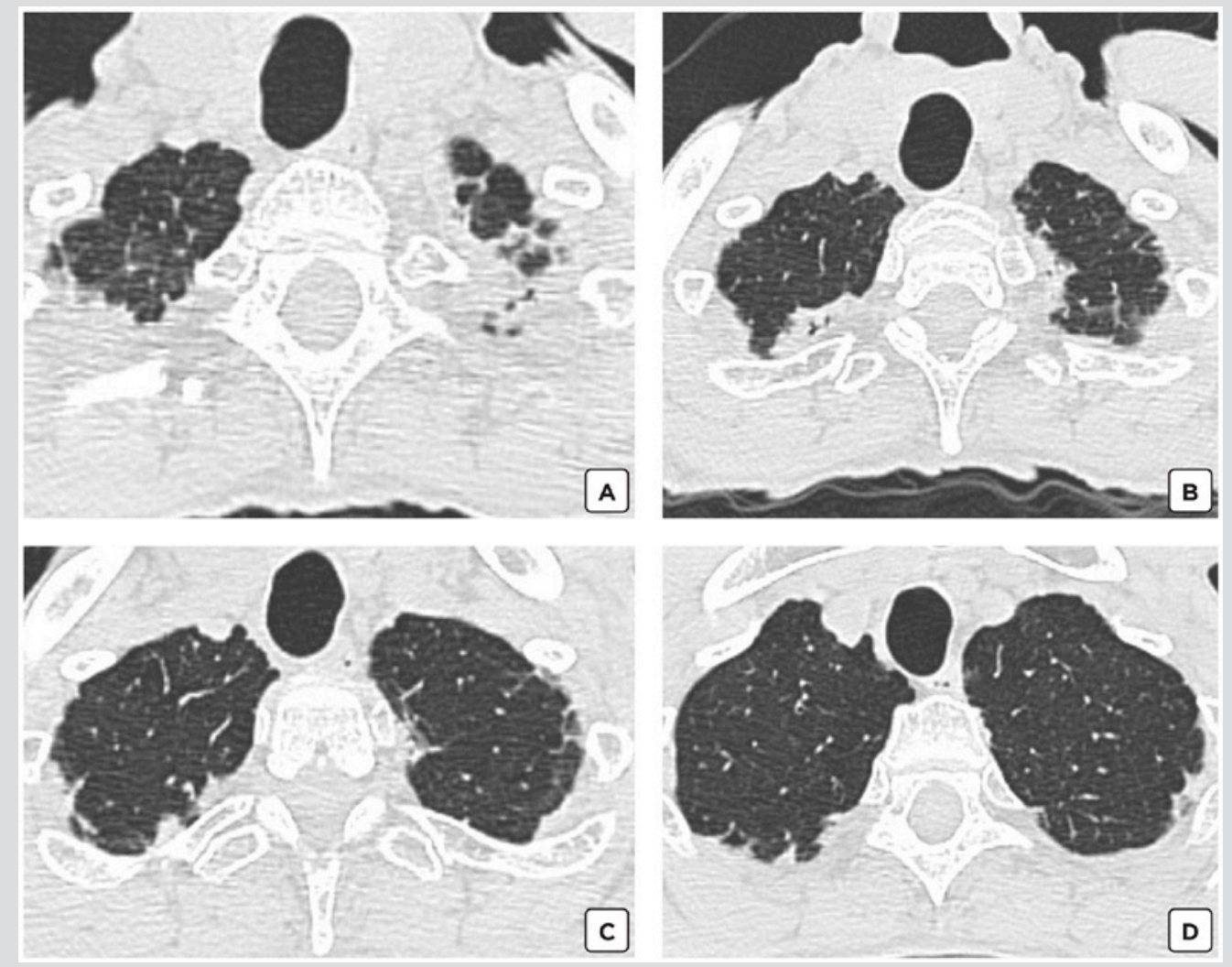

Figure 1. HRCT axial images showing fibrotic foci of the visceral pleura of the upper lobes. (a, b) Superior views; $(c, d)$ inferior views

She was referred to the pulmonology department for management of suspected ILD. Remarkably, the patient had no complaints of dyspnoea or cough. Her physical examination revealed an extremely low weight $\left(13.8 \mathrm{~kg} / \mathrm{m}^{2}\right)$, normal lung sounds and absence of platythorax or digital clubbing. Blood tests revealed mild anaemia and an elevated erythrocyte sedimentation rate ( $28 \mathrm{~mm} / \mathrm{h})$. The autoimmune panel was negative. Pulmonary function tests showed a preserved FVC ( $87 \%$ predicted) although with a moderate decline in DLCO (41\% predicted). At an ILD multidisciplinary group discussion, a provisional diagnosis of PPFE was assumed on the basis of clinical and radiological findings (typical HRCT pattern), with a surgical lung biopsy procedure deemed unnecessary. After exclusion of other possible underlying causes, a possible link with CD was considered. The patient was prescribed influenza and pneumococcal immunization and was started on low-dose immunomodulation with hydroxychloroquine $200 \mathrm{mg}$ once daily and prednisolone $2.5 \mathrm{mg}$ once daily.

The follow-up investigation at 12 months showed clinical and radiological stability and stable lung function.

\section{DISCUSSION}

In addition to idiopathic PPFE (e.g., the above-mentioned non-specific interstitial pneumonia), PPFE occurs as familial disease and also in association with several systemic conditions potentially affecting the immune system, such as stem cell or lung transplantation, rheumatoid arthritis, ankylosing spondylitis, radiation and anti-cancer chemotherapy, occupational dust exposure, hypersensitivity pneumonitis and infections ${ }^{[6]}$. 
This case is the first report of recognized PPFE in the context of CD. Regarding inflammatory bowel disease, an apical fibrocystic disease pattern has already been reported in ulcerative colitis, although it does not clearly resemble PPFE on HRCT [5, 6]. In our patient, a possible differential diagnosis would be drug-induced lung toxicity or other ILDs already reported to occur primarily in CD. Although the imaging pattern was not consistent with mesalazine-induced lung disease, which commonly presents as pulmonary infiltrates with eosinophilia, there was no blood eosinophilia and there was no sustained progression under maintenance treatment with mesalazine.

In addition, to the best of our knowledge, there are no case reports of PPFE secondary to azathioprine and also the time lapse was not consistent with this possible aetiology. Furthermore, the HRCT pattern was not consistent with non-specific interstitial pneumonia or organizing pneumonia ${ }^{[5]}$.

The intestinal and respiratory epithelia have a common embryonic origin in the primitive foregut, are like submucosal lymphoid tissue and may both react similarly when exposed to antigens in a susceptible patient ${ }^{[3,5]}$. Therefore, the same mechanism underlying intestinal inflammation and consequent fibrosis could be involved in the lung, leading to a PPFE pattern ${ }^{[5,7,8]}$. Also, an autoimmune predisposition seems to be a trigger for the development of increased numbers of elastic fibres in lung tissue ${ }^{[7]}$.

The present case, in accordance with previous reports ${ }^{[9]}$, had a typical HRCT pattern allowing for a definite PPFE diagnosis ${ }^{[1]}$. Although the classic diagnosis of PPFE is based on histological findings of homogenous subpleural intra-alveolar fibrosis with alveolar septal elastosis sparing the parenchyma away from the pleura ${ }^{[10]}$, surgical biopsies have been noticeably associated with a higher risk of prolonged bronchopleural fistula and substantial morbidity in PPFE patients. Currently, pathological information should be sought only when deemed necessary for a confident diagnosis after multidisciplinary integration of clinical, immunological and radiological findings ${ }^{[1]}$.

The natural history of PPFE is very variable. Although some cases may show uncontrolled fibrotic progression eventually leading to chronic respiratory failure and death, some patients may demonstrate long-lasting stability. The management of PPFE remains a major challenge as there are no standard treatments ${ }^{[1,6]}$. Corticosteroids and immunosuppressants seem to offer transitory or no improvement ${ }^{[1,11]}$. Despite the lack of evidence-based guidance, in patients thought to have some degree of immune dysregulation, the use of low intensity immunomodulation has been advocated. Anecdotally, prophylactic antibiotics are sometimes used in patients with PPFE more predisposed to infection ${ }^{[1]}$. Respiratory rehabilitation is an important treatment strategy for symptomatic patients [1] and a lung transplant might be considered in severe eligible cases ${ }^{[11]}$.

To conclude, we report the first case of PPFE associated with CD. As such, this type of chronic lung injury should be included among the forms of ILD which are possibly direct extra-intestinal manifestations of CD. Future studies will be important to further investigate this pathological association and its prognostic implications.

\section{REFERENCES}

1. Chua F, Desai SR, Nicholson AG, Devaraj A, Renzoni E, Rice A, et al. Pleuroparenchymal fibroelastosis - a review of clinical, radiological, and pathological characteristics. Ann Am Thorac Soc 2019;6(11):1351-1359.

2. Martins CT, Gonçalves JR. Fronteiras do pulmão - relação com o sistema gastroenterológico. Rev Port Pneumol 2020;16(1).

3. Mahadeva R, Walsh G, Flower CDR, Shneerson JM. Clinical and radiologic characteristics of lung disease in inflammatory bowel disease. Eur Respir J 2000;15:41-48.

4. Majewski S. Pulmonary manifestations of inflammatory bowel disease. Arch Med Sci 2015;11(6):1179-1188.

5. Yilmaz A, Demirci NY, Hoşgün D, Üner E, Erdoğan Y, Gökçek A, et al. Pulmonary involvement in inflammatory bowel disease. World J Gastroenterol 2010;16(39):4952-4957.

6. Singh R, Sundaram P, Joshi JM. Upper lobe fibrosis in ulcerative colitis. J Assoc Physicians India 2003;51:515-517.

7. Kinoshita Y, Watanabe K, Ishii H, Kushima H, Hamasaki M, Fujita M, et al. Pleuroparenchymal fibroelastosis as a histological background of autoimmune diseases. Virchows Archiv 2019;474(1):97-104.

8. Rogler G, Hausmann M. Factors promoting development of fibrosis in Crohn's disease. Front Med 2017;4:96.

9. Reddy TL, Tominaga M, Hansell DM, von der Thusen J, RassI D, Parfrey H, et al. Pleuroparenchymal fibroelastosis: a spectrum of histopathological and imaging phenotypes. Eur Respir J 2012;40(2):377-385.

10. Cheng SKH, Chuah KL. Pleuroparenchymal fibroelastosis of the lung: a review. Arch Pathol Lab Med 2016;140(8):849-853.

11. Camus P, von der Thüsen J, Hansell DM, Colby TV. Pleuroparenchymal fibroelastosis: one more walk on the wild side of drugs? Eur Resp J 2014;44:289-296. 